\title{
Several Effective Measures for Minus Excess Mortality of COVID-19 in Japan Including Mutual Interrelationships and Long-Term Care Facilities (LTCF)
}

\author{
Hiroshi Bando ${ }^{1,2^{*}}$ \\ ${ }^{1}$ Medical Research/Tokushima University, Tokushima, Japan \\ ${ }^{2}$ Integrative Medicine Japan (IMJ), Shikoku Island division, director, Tokushima, Japan
}

Corresponding Author: Hiroshi BANDO, MD, PhD, FACP ORCID iD

Address: Tokushima University /Medical Research, Nakashowa 1-61, Tokushima 770-o943, Japan; Email:pianomed@bronze.ocn.ne.jp

Received date: 14 October 2021; Accepted date: og November 2021; Published date: 16 November 2021

Citation: Bando H. Several Effective Measures for Minus Excess Mortality of COVID-19 in Japan Including Mutual Interrelationships and Long-Term Care Facilities (LTCF). Asp Biomed Clin Case Rep. 2021 Nov 16;4(3):191-94.

Copyright (C) 2021 Bando H. This is an open-access article distributed under the Creative Commons Attribution License, which permits unrestricted use, distribution, and reproduction in any medium provided the original work is properly cited.

\section{Abstract}

The impact of COVID-19 can be shown by life expectancy, excess death and total years of life lost (YLL). United States showed life expectancy minus 1.67 years, excess deaths 375,235 and total YLL 7,362,555. The excess death of Japan has remained minus value for long, in which long-term care facilities (LTCF) may contribute. LTCF has characteristic points as i) mutual interrelationships between hospitals, medical societies and prefectural offices, ii) rapid communication channels for regulatory official authorities, iii) high degree of citizenship and cooperation of all Japanese people for daily life and iv) mild lockdown without any punishment with declaration.

\section{Keywords}

COVID-19, Excess Death, Years of Life Lost (YLL), Long-Term Care Facilities (LTCF), National Institute of Infectious Disease (NIID), Excess Mortality

\section{Abbreviations}

YLL: Years of Life Lost; LTCF: Long-Term Care Facilities; NIID: National Institute of Infectious Disease

COVID-19 pandemic has been a crucial medical and social problem. Various statistic research worldwide has been reported [1]. Among them, authors presented reports from excess death rate, lifestyle, and integrative medicine (IM) [2]. The excess death in Japan has been minus value and remained extremely low [3]. This continuation for long was evaluated to be rare [4]. The estimation model period was studied using 2005-2020 data concerning the Japanese pneumococcal vaccine and ICD-10 by WHO [5]. Consequently, significantly negative excess mortality in
Japan seems to be from long-term people's precautions, such as maintaining social distancing, washing hands with alcohol, and wearing masks. These behaviors seemed to reduce the risk of pneumonia of COVID-19 and others.

For western countries, excess mortality was investigated [6]. Related data were available from the database of EUROSTAT which is the statistical office of the European Union (EU). Some factors including agespecific mortality rate in 2000, 2010, 2017 in the US 
were compared to those of European countries [7]. These data will become reference data for successive research concerning COVID-19 in the future. The impact of COVID-19 can be shown by 3 metrics, which are life expectancy, excess deaths, and total years of life lost (YLL). The results showed life expectancy minus 1.67 years, excess deaths 375,235 (attribution direct $83 \%$, indirect $17 \%$ ) and total YLL 7,362,555 for USA (direct 73\%, indirect 27\%). These results suggested considerable heterogeneity for the individual level [8]. To understand the mortality of COVID-19, it is important to analyze how earlier the death occurs. The data of YLL were calculated from 81 countries related to COIVD-19 as well as estimated excess deaths [9]. As a result, the loss of 20.5 million YLL was supposed across the world. On Jan 6, 2021, YLL of countries with much influence of COVID-19 showed 29 times of seasonal influenza. Furthermore, 3/4 of lost YLL is from the death of $<75$ years old, $1 / 3$ is from $<55$ years, and men have $45 \%$ more than women.

Unlike other countries, no excess mortality of COVID-19 was shown by the statistics throughout Japan [10]. The study was performed using the research model of the National Institute of Infectious Disease (NIID). There are some peak waves of infection, then excess mortality will be checked in detail in the future. The mortality and morbidity rates of COVID-19 were studied in 14 prefectures of Japan [11]. Each prefecture should have more than 4 deaths and 10 cases per day. As a result, a significant correlation was found between population density and morbidity rate $(\mathrm{R} 2=0.394)$. For weather factors, lower mortality and morbidity are observed for higher temperature and absolute humidity. By multivariate analysis, the coefficient for the decay, spread, the combined stage was proved to be $0.785,0.708$, 0.615 , respectively.

COVID-19 pandemic has brought interruptions of treatment for non-communicable diseases (NCDs) and non-emergency problems [12]. It is reported that about $40 \%$ of adults in the US seem to avoid regular medical visits [13]. From surveys in 47 countries, only $14 \%$ of healthcare providers could provide usual style treatment as face-to-face situation [12]. The number of prescriptions for a month at the pharmacy has decreased by 25\% between May 2019 and May 2020
[14]. For departments of otorhinolaryngology and pediatrics, prescriptions decreased by 50\% [14]. Some reasons exist from clinics and patients. Several medical faculties rescheduled the patient's visits for nonemergency cases, to deal with emergent patients with COVID-19 [15]. Patients may refrain from visiting clinics or hospitals, because of the possible risk of receiving infection. Further, they cannot possibly visit clinics from financial impact by COVID-19 [16]. Such social and psychological aspects should be considered from various points of view [17].

For the occupational aspect, socioeconomic status was examined for the interruption of ordinary medical treatment [18]. Out of 9510 cases, $11 \%$ experienced treatment interruptions. The odds ratio (RO) showed not married 1.44, manual labor work/desk work 1.30, lost employment and continued unemployment 1.62 and 2.57, feeling unstable finance 2.92. Consequently, treatment interruption may be a health inequality. The impact of COVID-19 on healthcare access was evaluated regarding patient visits. As a result, limited access showed the crucial function of long-term monitoring of vulnerable people and the urgent necessity of supporting medical facilities in the future [19].

Lockdown measures have been continued in many countries associated with several restrictions and punishments. On the other hand, Japan conducted mild lockdown, which was not enforceable or non-punitive with the declaration [20]. Between Japan and western countries, the difference has been suggested concerning the results of COVID-19. The initial and consecutive response to the pandemic was similar. The number of deaths, however, was different and very low in Japan. One of the reasons would be the existence of many long-term care facilities (LTCF) in Japan [21]. Unlike hospitals that are in charge of emergency care, LTCF are facilities that provide consecutive care for elderly people [22]. In LTCFs, a remarkably lower incidence of COVID-19 has been observed in comparison with those in other countries [23].

For LTCFs in Japan, some characteristic and beneficial points have been found [23]. They are i) LTCFs have mutual interrelationships between 
Citation: Bando H. Several Effective Measures for Minus Excess Mortality of COVID-19 in Japan Including Mutual Interrelationships and Long-Term Care Facilities (LTCF). Asp Biomed Clin Case Rep. 2021 Nov 16;4(3):191-94.

hospitals, medical societies, and prefectural offices for long years, ii) especially for managements of COVID19, effective and rapid communication channels have been always continued between LTCFs and regulatory official authorities, iii) such authorities include each prefectural office and health center, medical societies and media relations, iv) successful routine managements and protocols for control and prevention of COVID-19 have been continued in LTCFs, v) perfect understanding and cooperation of families and related personnel of the patient in LTCFs, vi) high degree of citizenship and cooperation and implementation of all Japanese people for daily life and vii) no official or governmental lockdown is present in Japan without any punishment or crime, but people can continue restricted daily life for long leading to safe and happiness of other people [24].

\section{Conflict of Interest}

The author has read and approved the final version of the manuscript. The author has no conflicts of interest to declare.

\section{References}

[1] Karlinsky A, Kobak D. The World Mortality Dataset: Tracking excess mortality across countries during the COVID-19 pandemic. medRxiv [Preprint]. 2021 Jun 4:2021.01.27.21250604. Update in: Elife. 2021 Jun 30;10: [PMID: 33532789]

[2] Bando H. Fewer Annual Deaths in Japan with Negative Excess Mortality for COVID-19. Asp Biomed Clin Case Rep. 2021 Apr 06;4(1):84-87.

[3] Woolf SH, Chapman DA, Sabo RT, Zimmerman EB. Excess Deaths from COVID-19 and Other Causes in the US, March 1, 2020, to January 2, 2021. JAMA. 2021 Apr 2;325(17):1786-89. [PMID: 33797550]

[4] Bando H. Some Measures for COVID-19 Including Deep Ultraviolet Light-Emitting Diode (DUV-LED), Gc protein-derived Macrophage-Activating Factor (Gcmaf), and 5-Aminolevulinic Acid (5-ALA). Asp Biomed Clin Case Rep. 2021 Jun 30;4(2):110-13.

[5] Kurita J, Sugawara T, sugishita Y, Ohkusa Y. Negative Excess Mortality in Pneumonia Death caused by COVID-19 in Japan. medRxiv. 2021 Jan 22;21250283.

[6] Bogos K, Kiss Z, Kerpel Fronius A, Temesi G, Elek J, Madurka I, Cselkó Z, Csányi P, Abonyi-Tóth Z, Rokszin
G, Barcza Z, Moldvay J. Different Trends in Excess Mortality in a Central European Country Compared to Main European Regions in the Year of the COVID-19 Pandemic (2020): a Hungarian Analysis. Pathol Oncol Res. 2021 Apr 13;27:16o9774. [PMID: 34257618]

[7] Preston SH, Vierboom YC. Excess mortality in the United States in the 21st century. Proc Natl Acad Sci U S A. 2021 Apr 20;118(16):e2024850118. [PMID: 33846260 ]

[8] Chan EYS, Cheng D, Martin J. Impact of COVID-19 on excess mortality, life expectancy, and years of life lost in the United States. PLoS One. 2021 Sep 1;16(9):eo256835. [PMID: 34469474]

[9] Pifarré I Arolas H, Acosta E, López-Casasnovas G, Lo A, Nicodemo C, Riffe T, Myrskylä M. Years of life lost to COVID-19 in 81 countries. Sci Rep. 2021 Feb 18;11(1):3504. Erratum in: Sci Rep. 2021 Apr 14;11(1):8543. [PMID: 33603008]

[10] Kurita J, Sugawara T, Ohkusa Y. Excess Mortality Probably Attributable to COVID-19 in Tokyo, Japan During August and October 2020. J Disaster Res. 2021;16(5):890-94.

[11] Kodera S, Rashed EA, Hirata A. Correlation between COVID-19 Morbidity and Mortality Rates in Japan and Local Population Density, Temperature, and Absolute Humidity. Int J Environ Res Public Health. 2020 Jul 29;17(15):5477. [PMID: 32751311]

[12] Chudasama YV, Gillies CL, Zaccardi F, Coles B, Davies MJ, Seidu S, Khunti K. Impact of COVID-19 on routine care for chronic diseases: A global survey of views from healthcare professionals. Diabetes Metab Syndr. 2020 Sep-Oct;14(5):965-67. [PMID: 32604016] [13] Czeisler MÉ, Marynak K, Clarke KEN, Salah Z, Shakya I, Thierry JM, Ali N, McMillan H, Wiley JF, Weaver MD, Czeisler CA, Rajaratnam SMW, Howard ME. Delay or Avoidance of Medical Care Because of COVID-19-Related Concerns - United States, June 2020. MMWR Morb Mortal Wkly Rep. 2020 Sep 11;69(36):1250-57. [PMID: 32915166]

[14] LoPresti M, Seo T, Sato N. Pandemics and access to care: use of real-world DATA to examine the IMPACT of COVID-19 on pharmacy visits in Japan. Value Health. 2020 Dec;23:S685.

[15] Chopra V, Toner E, Waldhorn R, Washer L. How Should U.S. Hospitals Prepare for Coronavirus Disease 2019 (COVID-19)? Ann Intern Med. 2020 May 5;172(9):621-22. [PMID: 32160273] 
Citation: Bando H. Several Effective Measures for Minus Excess Mortality of COVID-19 in Japan Including Mutual Interrelationships and Long-Term Care Facilities (LTCF). Asp Biomed Clin Case Rep. 2021 Nov 16;4(3):191-94.

[16] McMaughan DJ, Oloruntoba O, Smith ML. Socioeconomic Status and Access to Healthcare: Interrelated Drivers for Healthy Aging. Front Public Health. 2020 Jun 18;8:231. [PMID: 32626678]

[17] Saunders CL, Flynn S, Massou E, Lyratzopoulos G, Abel G, Burt J. Sociodemographic inequalities in patients' experiences of primary care: an analysis of the General Practice Patient Survey in England between 2011 and 2017. J Health Serv Res Policy. 2021 Jul;26(3):198-207. [PMID: 33517786]

[18] Fujimoto K, Ishimaru T, Tateishi S, Nagata T, Tsuji M, Eguchi H, Ogami A, Matsuda S, Fujino Y; CORoNaWork Project. A cross-sectional study of socioeconomic status and treatment interruption among Japanese workers during the COVID-19 pandemic. J Occup Health. 2021 Jan;63(1):e12232. [PMID: 33998105]

[19] Makiyama K, Kawashima T, Nomura S, Eguchi A, Yoneoka D, Tanoue Y, Kawamura Y, Sakamoto H, Gilmour S, Shi S, Matsuura K, Uryu S, Hashizume M. Trends in Healthcare Access in Japan during the First Wave of the COVID-19 Pandemic, up to June 2020. Int J
Environ Res Public Health. 2021 Mar 22;18(6):3271. [PMID: 33809955]

[20] Sugaya N, Yamamoto T, Suzuki N, Uchiumi C. A real-time survey on the psychological impact of mild lockdown for COVID-19 in the Japanese population. Sci Data. 2020 Oct 29;7(1):372. [PMID: 33122626]

[21] Estévez-Abe M, Ide H. COVID-19 and Long-Term Care Policy for Older People in Japan. J Aging Soc Policy. 2021 Jul-Oct;33(4-5):444-58. [PMID: 33988089]

[22] Takehisa Y. Investigation for medical and longterm care in Japan. International Medicine 2021; 3(2): 28-30.

[23] Estévez-Abe M, Ide H. COVID-19 and Japan's Small Death Toll in Long-Term Care Facilities. Journal of Aging and Social Policy. Weatherhead Center for International Affairs (WCFFIA), Harvard University; 2021.

[24] Bando H. Lifestyle improvement with the philosophy of Hinohara-ism in the current challenging circumstances of COVID-19 worldwide. Clin Med Case Rep 2021;5:e101.

Keywords: COVID-19, Excess Death, Years of Life Lost (YLL), Long-Term Care Facilities (LTCF), National Institute of Infectious Disease (NIID), Excess Mortality 